\title{
Harmonic Distortion in Fully-Differential Switched-Current Sigma-Delta Modulators
}

\author{
J.M. de la Rosa, B. Pérez-Verdú, F. Medeiro, R. del Río and A. Rodríguez-Vázquez \\ Instituto de Microelectrónica de Sevilla, IMSE-CNM (CSIC) \\ Edificio CICA-CNM, C/Tarfia s/n, 41012-Sevilla, SPAIN \\ Phone: +34 95 4239923; FAX: +34 954231832 \\ E-mail: jrosa@imse.cnm.es
}

\begin{abstract}
This paper presents a systematic analysis of the harmonic distortion in $\Sigma \Delta$ modulators ( $\Sigma \Delta \mathrm{Ms})$ implemented with fully-differential switched-current (SI) circuits. Closed form expressions are derived for the third-order harmonic distortion in both lowpass and bandpass $\Sigma \Delta \mathrm{Ms}$. For the latter, the third-order intermodulation distortion is also deduced. Time domain behavioral simulations validate our approach. ${ }^{(*)}$
\end{abstract}

\section{Introduction}

The trend towards the realization of mixed-signal systems on chip has motivated exploring analog design techniques compatible with standard, digital CMOS technologies. This is the case of switched-current circuits (SI) [1], which during the last ten years have been used for different analog functions, including filtering [2] and A/D conversion [3]. Particularly, several SI $\Sigma \Delta \mathrm{Ms}$ have been reported, for lowpass signals [4] [5], as well as for bandpass signals [6].

Performances reported to date for SI $\Sigma \Delta \mathrm{Ms}$ are lower than for state-of-the-art SC $\Sigma \Delta \mathrm{Ms}$ [7]. For instance, [5] obtains 13-bit for lowpass voice band signals, while [6] obtains 9-bits for bandpass signals in the AM bandwidth. Among other reasons, such lower performances are motivated by larger influence of SI non-idealities, as compared to SC ones, and by incomplete modeling of their influence [8][9][10][11]. Particularly, harmonic distortion is recognized as one of the most important SI performance-degrading nonlinearities.

Error mechanisms responsible for harmonic distortion include: threshold voltage mismatch, non-linear finite output-input conductance ratio, charge injection, and settling error. Their influence on memory cell distortion has been analysed elsewhere [8][9]. However, only the charge injection error analysis has been extended to lowpass SI $\Sigma \Delta \mathrm{Ms}[10]$. Based on the harmonic distortion analysis of SI blocks, this paper presents closed-form equations for third-order distortion coefficients of SI integrators, resonators and both lowpass and bandpass $\Sigma \Delta \mathrm{Ms}$. The analyses presented here have been validated by time-domain behavioral simulations [11] and are illustrated through results pertaining to the harmonic distortion due to charge injection and non-linear output-input conductance ratio.

${ }^{(*)}$ This work has been supported by the Spanish CICYT Project TIC 97-0580 and the ESPRIT Project 29621.

\section{Non-linear modeling of the memory cell opera- tion}

Fig.1 shows a simple, second-generation memory cell. Ideally, the output current is a half-delayed inverted version of the input ${ }^{\dagger}$

$$
i_{o, n}=-i_{i, n-1 / 2}
$$

According to [1], its main non-idealities are: finite output-input conductance ratio error (represented by $\left.\varepsilon_{g}\right)$, incomplete settling error $\left(\varepsilon_{s}\right)$, charge injection error $\left(\varepsilon_{q}\right)$ and thermal noise. In the presence of these errors, the output current can be generically expressed as

$i_{o, n}=I_{o f f}-\left(1-\xi_{1}\right) i_{i, n-1 / 2}+i_{t h}+i_{H}\left(i_{i, n-1 / 2}\right)(2)$ where $I_{\text {off }}$ stands for the offset current at the output, $\xi_{1}$ is the linear gain error, $i_{t h}$ is the thermal noise contribution and $i_{H}$ represents the non-linearity.

We will assume that the memory cell reaches the steady state before the end of the sampling phase and, consequently, settling error $\varepsilon_{s}$ will not be considered. Besides, thermal noise and offset current, will not be included in our analysis because they do not contribute to the harmonic distortion. Regarding $i_{H}$, it will be expressed as a polynomial function of the input current $i_{i}$ [8][9]

$$
i_{H}=-\sum_{k=2}^{\infty} \xi_{k} i_{i, n-1 / 2}^{k}
$$

where $\xi_{k}$ have different expressions for each error.

Taking into account the above considerations, expression (2) can be simplified into,

$$
i_{o, n}=-\left(1-\xi_{1}\right) i_{i, n-1 / 2}-\sum_{k=2}^{\infty} \xi_{k} i_{i, n-1 / 2}^{k}
$$

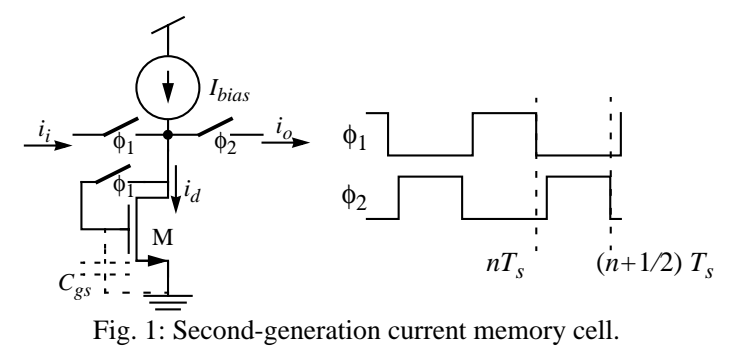

$\dagger$. We use the notation $i_{\mathrm{o}, n}$ to represent $i_{\mathrm{o}}\left(n T_{s}\right)$, where $T_{s}$ is the sampling period. 
For illustration purposes, Table 1 shows $\xi_{1}, \xi_{2}$ and $\xi_{3}$ when the memory cell is degraded by charge injection [9], and non-linear output-input conductance ratio error. For the latter, it has been assumed that the memory transistor transconductance depends on the input signal as $g_{m}=g_{m Q} \sqrt{1+i_{i} / I_{\text {bias }}}$, with $g_{m O}$ being the operating-point transconductance. In Table $1,\left(V_{g s}-V_{T}\right)_{Q}$ represents the quiescent excess overdrive voltage; ${ }^{2} V_{\text {off }}$ is the offset introduced by the charge injected; $C_{s w}$ is the memory switch capacitance; $g_{\text {out }}$ is the output conductance and $A_{V}$ is the gain of the amplifier stage used either to reduce the output conductance (in regulated-cascode memory cells), or to increase the input conductance (in folded regulated-cascode memory cells). Note that $A_{V}=1$ for the simple memory cell.

Assuming that the input current of the memory cell in Fig. 1 is a sinusoidal signal of amplitude $I_{i}$, the output current will contain harmonics of the input signal frequency $f_{i}$. The $k$-order harmonic distortion, $H D_{k}$, is defined as the ratio of the output signal amplitude at frequency $k f_{i}$ to the linear output amplitude. For our analysis, we will assume fully-differential memory cells. Thus, even powers of the input current in (4) can be considered negligible. On the other hand, assuming that the third-order harmonic is dominant, (4) can be simplified into

$$
i_{o, n} \cong-\left(1-\xi_{1}\right) i_{i, n-1 / 2}-\xi_{3} i_{i, n-1 / 2}^{3}
$$

In this case, the Total Harmonic Distortion (THD) is approximately equal to $H D_{3}$ and given by

$$
T H D \cong H D_{3}=\frac{\xi_{3}}{4\left(1-\xi_{1}\right)} I_{i}^{2}
$$

\section{Harmonic distortion in fully-differential SI inte- grators}

Fig. 2 shows the schematic of a fully-differential LDI SI integrator. It is composed of two cells, and an output stage. In the following, it will be assumed that the operation of the memory cells is described by (5). Although these memory cells are simple, our analysis can be extended to enhanced memory cells - cascode, regulated-cascode or folded regulated-cascode - by conveniently changing the expressions of $\xi_{1}$ and $\xi_{3}$.

The operation of the integrator is as follows. After clock phase $\phi_{1}$, which goes on for $n T_{s}$, the differen-

Table 1: $\xi_{1}, \xi_{2}$ and $\xi_{3}$ for some errors in the memory cell.

\begin{tabular}{|l|c|c|}
\hline & $\varepsilon_{g}$ & $\varepsilon_{q}$ \\
\hline$\xi_{1}$ & $\frac{g_{\text {out }}\left(V_{g s}-V_{T}\right)}{A_{V} I_{\text {bias }}}$ & $\frac{-C_{s w} / C_{g s}\left(V_{T}+2\left(V_{g s}-V_{T}\right)\right.}{\left(V_{g s}-V_{T}\right)-V_{\text {off }}}$ \\
\hline$\xi_{2}$ & $\frac{g_{\text {out }}\left(V_{g s}-V_{T}\right)}{2 A_{V} I_{\text {bias }}^{2}}$ & $\frac{-V_{T}\left(C_{s w} / C_{g s}\right)-V_{\text {off }}}{4 I_{\text {bias }}\left(V_{g s}-V_{T}\right)}$ \\
\hline$\xi_{3}$ & $\frac{-3 g_{\text {out }}\left(V_{g s}{ }^{\left.-V_{T}\right)} Q\right.}{8 A_{V} I_{\text {bias }}^{3}}$ & $\frac{V_{T}\left(C_{\text {sw }} / C_{g s}\right)+V_{\text {off }}}{8 I_{\text {bias }}^{2}\left(V_{g s}-V_{T}\right)}$ \\
\hline
\end{tabular}

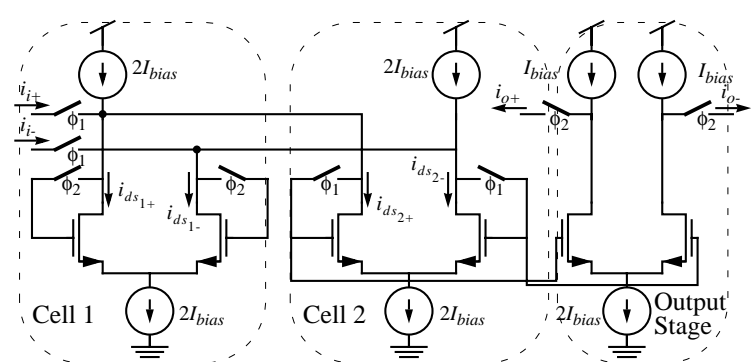

Fig.2. Fully-Differential LDI SI integrator.

tial drain current of the memory cell 2 , is given by

$$
\begin{gathered}
i_{d s_{2, n}} \equiv i_{d s_{2+, \mathrm{n}}}-i_{d s_{2-\mathrm{n}}} \cong \\
\cong\left(1-\xi_{1}\right)\left(i_{i, n}-i_{d s_{1, n-1 / 2}}\right)+\xi_{3}\left(i_{i, n}-i_{d s_{1, n-1 / 2}}\right)^{3}
\end{gathered}
$$

where $i_{d s_{1, n}} \equiv\left(i_{d s_{1+\mathrm{n}}}-i_{d s_{1-\mathrm{n}}}\right)$ represents the differential drain current of memory cell 1 .

After clock phase $\phi_{2}$,

$$
i_{d s_{1, n+1 / 2}} \cong\left(1-\xi_{1}\right)\left(-i_{d s_{2, n}}\right)+\xi_{3}\left(-i_{d s_{2, n}}\right)^{3}
$$

Assuming that the output stage (represented in Fig. 2 as a simple current mirror) is ideal, the output current of the integrator is given by

$$
i_{o, n+1 / 2}=-i_{d s_{2, n}}
$$

From (7), (8) and (9) it can be derived that the output current of the integrator is

$$
i_{o, n} \cong\left(1-\xi_{1}\right) i_{x, n}+\xi_{3} i_{x, n}^{3}
$$

where

$$
i_{x, n}=-i_{i, n-1 / 2}+\left(1-\xi_{1}\right) i_{o, n-1}+\xi_{3} i_{o, n-1}^{3}
$$

Assuming that $\xi_{1}, \xi_{3}\left|i_{o, n}\right|^{2} \ll 1$ and performing a Taylor series expansion of (10), obtains

$$
i_{o, n} \cong\left(1-2 \xi_{1}\right) i_{o, n-1}-\left(1-\xi_{1}\right) i_{i, n}^{*}
$$

where

$$
i_{i, n}^{*} \cong i_{i, n-1 / 2}-\frac{\xi_{3}}{\left(1-\xi_{1}\right)}\left(i_{o, n-1}^{3}+i_{o, n}^{3}\right)
$$

Thus, the analysis of an SI integrator formed by memory cells with non-linear errors can be accomplished considering an integrator formed by memory cells with linear gain errors whose input signal is equal to (13). The equivalent distortion at the integrator input can be estimated by analysing the harmonic content of such an expression.

For this purpose, let assume that the input current is a sinusoidal signal of amplitude $I_{i}$ and frequency $f_{i}$. In this case, the output current of the integrator will be a periodic signal, being the amplitude of its fundamental harmonic approximately given by

$$
\left.I_{o} \cong I_{i}\left|\frac{-\left(1-\xi_{1}\right) z^{-1 / 2}}{1-\left(1-2 \xi_{1}\right) z^{-1}}\right|\right|_{z=e^{j 2 \pi f_{i} T_{s}}}
$$


On the other hand, we will suppose that the output of the integrator can be approximated by its first harmonic, so that

$$
i_{o, n} \cong I_{o} \cos \left(2 \pi f_{i} n T_{s}\right)
$$

where $T_{s}$ is the sampling period. Substituting (14) and (15) in (12) and performing a Fourier series expansion, it can be shown that the third-order harmonic is

$$
\begin{aligned}
i_{3 f_{i}, n} \cong & \frac{\xi_{3} I_{o}^{3}}{4\left(1-\xi_{1}\right)}\left[\cos \left(6 \pi f_{i} n T_{s}\right)+\right. \\
& \left.+\cos \left(6 \pi f_{i}(n-1) T_{s}\right)\right]
\end{aligned}
$$

The amplitude of the third-order harmonic at the integrator input derived from (16) is

$$
A_{H, 3} \equiv\left|i_{3 f_{i}, n}\right| \cong \frac{\xi_{3} I_{o}^{3}}{2\left(1-\xi_{1}\right)}\left|\cos \left(3 \pi f_{i} T_{s}\right)\right|
$$

The amplitude of the third-order harmonic referred to the integrator output can be obtained by multiplying (17) by the module of the integrator transfer function

$$
A_{H, 3}^{\text {out }}=\frac{\xi_{3} I_{o}^{3}}{4\left(1-\xi_{1}\right)}\left|\cot \left(3 \pi f_{i} T_{s}\right)\right|
$$

The third-order harmonic distortion referred to the integrator output is calculated by dividing the above expression by $I_{o}$, giving

$$
H D_{3} \equiv \frac{A_{H, 3}^{\text {out }}}{I_{o}}=\frac{\xi_{3} I_{i}^{2}}{16\left(1-\xi_{1}\right)}\left|\frac{\cot \left(3 \pi f_{i} T_{s}\right)}{\left[\sin \left(\pi f_{i} T_{s}\right)\right]^{2}}\right|
$$

The above expression has been derived for the general case and hence, it can be used to predict the harmonic distortion in SI fully-differential integrators due to any error except for the settling.

As an application, let assume that the integrator in Fig. 2 is ideal except for the charge injection error. The theoretical prediction of the third-order harmonic distortion for this integrator is computed by substituting the corresponding expressions of $\xi_{1}$ and $\xi_{3}$ (see Table 1$)^{\dagger \dagger}$ in (19)

Fig.3 plots $\mathrm{HD}_{3}$ vs. $\mathrm{C}_{s w} / C_{g s}$ and compares the theoretical model with time-domain simulations using the SI behavioral simulator described in [11]. In this example: $\left(V_{g s}-V_{T}\right)_{Q}=0.1 \mathrm{~V}, V_{o f f}=0$ (commom mode signal), $V_{T}=1 \mathrm{~V}$ and $I_{\text {bias }}=200 \mu \mathrm{A}$. Note that, both the linear and the non-linear gain errors increase with $C_{s w} / C_{g s}$. Their effects on the harmonic distortion are well predicted by the model. On the other hand, the influence of the integrator gain is also considered by changing the input signal frequency. Several values of $f_{i}$ have been applied showing a good agreement with simulations.

$\dagger \uparrow$. These expressions correspond to a single-ended memory cell. For the case of fully-differential, $\xi_{1}$ has the same value and $\xi_{3} \rightarrow \xi_{3} / 4$

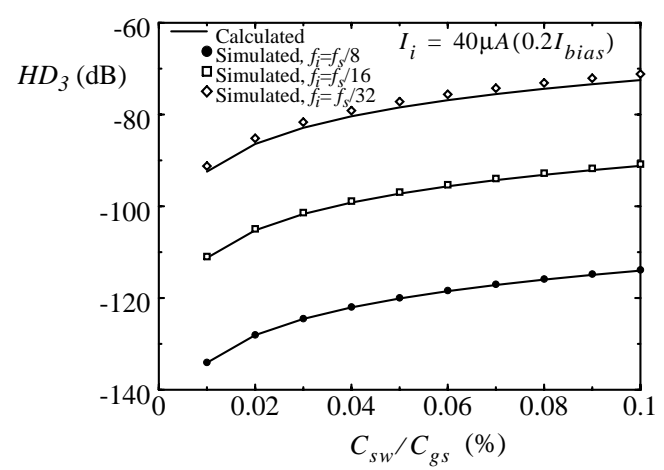

Fig. 3: Third-order harmonic distortion in Fully-Differential LD SI integrators due to non-linear $\varepsilon_{q} . H D_{3}$ vs. $C_{s w} / C_{g s}$.

\section{Harmonic distortion in SI lowpass $\Sigma \Delta$ modula- tors}

Fig. 4 shows the block diagram of a second-order lowpass $\Sigma \Delta$ modulator (2ndLP $\Sigma \Delta \mathrm{M}$ ) based on LDI integrators. Modelling the quantizer as an additive white noise source $E(z)$ [12], the z-domain modulator output is given by

$$
Y(z)=S_{T F}(z) X(z)+N_{T F}(z) E(z)
$$

where $X(z)$ and $Y(z)$ represent the input and the output of the modulator. In the ideal case, the $\underline{\text { Signal }}$ Transfer Function $\left(S_{T F}(z)\right)$ and the quantization Noise Transfer Function $\left(N_{T F}(z)\right)$ are respectively given by

$$
S_{T F}(z)=z^{-1} \quad N_{T F}(z)=\left(1-z^{-1}\right)^{2}
$$

For our analysis, the following considerations have been taken into account:

- The harmonic distortion referred to the modulator input is equal to the harmonic distortion referred to the modulator output. This is because the gain of $S_{T F}(z)$ is unity.

- The harmonic distortion referred to the first integrator input is added directly to the input signal. Thus, it is not attenuated in the base band. On the contrary, the contribution of the second integrator to the harmonic distortion is attenuated by the gain of the first integrator. For this reason, only the first integrator contribution has to be considered for the analysis.

Assuming that the transfer function of the first integrator is given by (14), it can be shown that the erroneous signal transfer function of a modulator like that shown in Fig.4 is given by

$$
S_{T F}^{e r r}(z) \cong \frac{\left(1-\xi_{1}\right) z^{-1}}{1+2 \xi_{1} z^{-1}+\xi_{1} z^{-2}}
$$

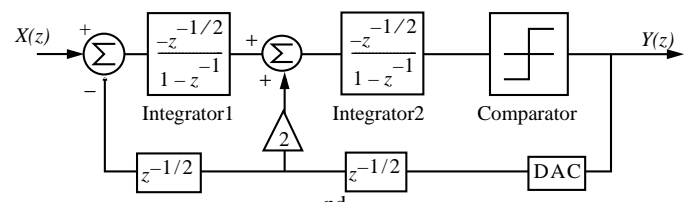

Fig. 4: Block diagram of the $2^{\text {nd }}$-order lowpass $\Sigma \Delta$ modulator. 
On the other hand, obviating the quantization noise, and assuming that $f_{i} T_{s} \ll 1$, the expressions for the first integrator input and output amplitudes are respectively given by

$$
\begin{gathered}
I_{i}(z) \cong 4 \pi\left(1-4 \xi_{1}\right) f_{i} T_{s} X(z) \\
I_{o} \cong \frac{I_{i}}{2 \pi f_{i} T_{s}}=2\left(1-4 \xi_{1}\right) X(z)
\end{gathered}
$$

Substituting (24) in (17) and dividing the result by the amplitude of the modulator input signal $A=|X(z)|$, obtains the third-order harmonic distortion at the modulator output as follows:

$$
H D_{3} \cong 4 \xi_{3}\left(1-11 \xi_{1}\right) A^{2}\left(1-\frac{3 \pi}{2 M}\right)
$$

where $M \equiv f_{s} /\left(2 f_{i}\right)$ represents the oversampling ratio.

The above expression has been validated by timedomain behavioral simulation. Fig.5 illustrates the harmonic distortion of the modulator in Fig.4 assuming that their memory cells are ideal except for the charge injection error. Fig.5(a) compares the predictions of (25) with simulations by plotting $\mathrm{HD}_{3}$ as a function of the ratio $C_{s w} / C_{g s}$. In this figure, the DAC reference current is $I_{D A C} \stackrel{g}{=} I_{\text {bias }} / 2$ while the input amplitude is $A=I_{D A C} / 2$. Fig. 5(b) shows a simulated output spectrum for $\left(V_{g s}-V_{T}\right)_{Q}=0.1 \mathrm{~V}$ and $C_{s w} / C_{g s}=0.08 \%$. The predicted data for the thirdorder harmonic distortion is $H D_{3}=-86 \mathrm{~dB}$ which agrees with the simulated data $\left(H D_{3}=-85 \mathrm{~dB}\right)$.
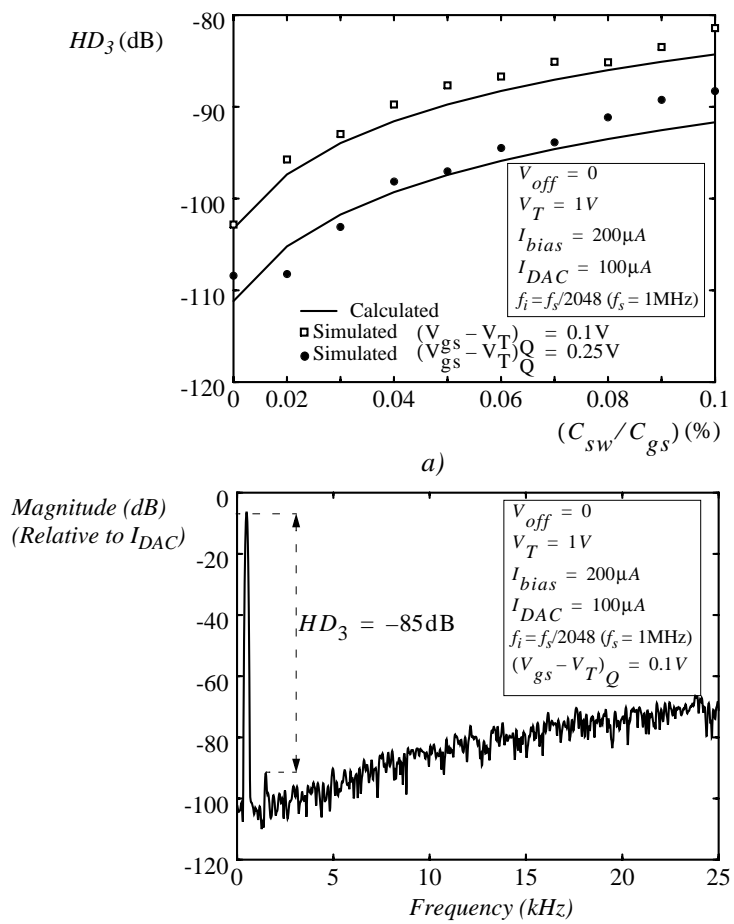

b)

Fig. 5: $H D_{3}$ at the output of a $2^{\text {nd }}$ order lowpass $\Sigma \Delta$ modulator due to $\varepsilon_{q}$. a) $H D_{3}$ vs. $C_{s w} / C_{g s}$ for different values of $\left(V_{g s}-V_{T}^{q}\right)$. b) Output spectrum corresponding to $\left(V_{g s}^{g s}-V_{T}^{T}\right)^{Q}=0.1 \mathrm{~V}$ and $C_{s w} / C_{g s}=0.08 \%$ (Input amplitude equal to $-6 \mathrm{~dB}$ DAC reference current $\left.I_{D A C}\right)$.

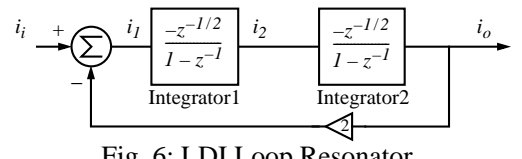

Fig. 6: LDI Loop Resonator.

\section{Harmonic distortion in SI bandpass $\Sigma \Delta$ modula- tors}

\section{A. Harmonic distortion in SI resonators}

Most of bandpass $\Sigma \Delta$ modulators reported in the literature obtain their architecture by applying the transformation $z^{-1} \rightarrow-z^{-2}$ to the corresponding lowpass $\Sigma \Delta$ modulators [12]. As a consequence of this transformation, the original integrators become resonators with a transfer function given by

$$
H(z)=\frac{z^{-a}}{1+z^{-2}}
$$

The value of $a$ depends on the transfer function of the original integrator. There are many filter structures which implement (26) [12]. Fig.6 shows the block diagram of one based on LDI integrators. This structure is advantageous as compared to the others because it remains stable under changes in the loop coefficients.

Let assume that the integrators which form the resonator in Fig. 6 are implemented as shown in Fig.2. In the presence of non-linear errors, they can be described by (12) and, hence, the difference equations which describe the behavior of the resonator are

$$
\begin{gathered}
i_{2, n} \cong-\left(1-\xi_{1}\right) i_{1, n-1 / 2}+\left(1-2 \xi_{1}\right) i_{2, n-1}+ \\
+\xi_{3} i_{2, n}^{3}+\xi_{3} i_{2, n-1}^{3} \\
i_{o, n} \cong-\left(1-\xi_{1}\right) i_{2, n-1 / 2}+\left(1-2 \xi_{1}\right) i_{o, n-1}+ \\
+\xi_{3} i_{o, n}^{3}+\xi_{3} i_{o, n-1}^{3}
\end{gathered}
$$

where $i_{1}$ and $i_{2}$ are respectively the input and the output of the first integrator in the loop (see Fig.6) while $i_{i}$ and $i_{o}$ are respectively the input and the output of the resonator.

Solving for $i_{2, n}$ in (28), substituting it in (27) and assuming that $\xi_{1}, \xi_{3}\left|i_{o, n}\right|^{2} \ll 1$, result in the following difference equation

$$
i_{o, n} \cong\left(1-2 \xi_{1}\right) i_{i, n}^{\prime}-\left(1-4 \xi_{1}\right) i_{o, n-2}
$$

where

$$
\begin{array}{r}
i_{i, n}^{\prime}=i_{i, n-1}+\frac{\xi_{3}}{1-2 \xi_{1}}\left[i_{o, n}^{3}-i_{o, n-2}^{3}+\right. \\
\left.\left(i_{o, n}-i_{o, n-1}\right)^{3}+\left(i_{o, n-1}-i_{o, n-2}\right)^{3}\right]
\end{array}
$$

Assuming that $i_{i}$ is a sinusoidal signal of amplitude $I_{i}, i_{o}$ will be a periodic signal, being the amplitude of its fundamental harmonic

$$
\left.I_{o} \cong I_{i}\left|\frac{\left(1-2 \xi_{1}\right) z^{-1}}{1+\left(1-4 \xi_{1}\right) z^{-2}}\right|\right|_{z=e^{j 2 \pi f_{i} T_{s}}}
$$




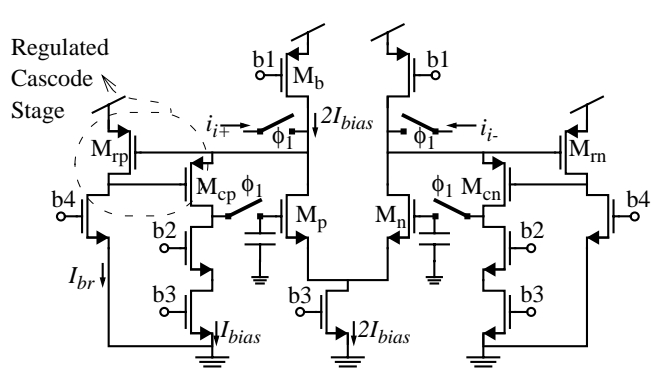

Fig. 7: Fully-differential regulated folded-cascode memory cell.

As for the case of the integrator, the output current of the resonator can be approximated by its fundamental harmonic, which can be generically expressed as

$$
i_{o, n} \cong I_{o} \cos \left(\phi_{n}\right)
$$

where $\phi_{n}=2 \pi f_{i} T_{s} n+\Phi\left(f_{i}, \xi_{1}\right)$ and $\Phi\left(f_{i}, \xi_{1}\right)$ is the phase delay caused by the resonator.

Substituting (32) in (30), and performing a Fourier series expansion, it can be shown that the third-order harmonic is

$$
\begin{aligned}
i_{3 f_{i}, n} \cong & \frac{-\xi_{3} I_{o}^{3}}{1-2 \xi_{1}}\left[\left(\frac{1}{2}+6 \pi f_{i}^{\prime} T_{s}\right) \cos \left(3 \phi_{n}\right)+\right. \\
& \left.+\left(3 \pi f_{i}^{\prime} T_{s}\right) \sin \left(3 \phi_{n}\right)\right]
\end{aligned}
$$

where $f_{i}^{\prime}=f_{i}-f_{s} / 4$, and $f_{i}^{\prime} T_{s} \ll 1$ has been assumed.

From (33) it can be derived that the amplitude of the third-order harmonic at the resonator input is

$$
A_{H, 3} \equiv\left|i_{3 f_{i}, n}\right| \cong \frac{\xi_{3} I_{o}^{3}}{2\left(1-2 \xi_{1}\right)}\left(1+12 \pi f_{i}^{\prime} T_{s}\right)
$$

Following the same procedure as in Section 3, the third-order harmonic distortion at the resonator output can be found by multiplying (34) by the resonator gain and dividing this result by $I_{o}$. This gives

$$
H D_{3} \cong \frac{\xi_{3} I_{i}^{2}}{384\left(1-2 \xi_{1}\right)\left(\pi f_{i}^{\prime} T_{s}\right)^{3}\left(1+\chi^{2}\right) \sqrt{1+\frac{\chi^{2}}{9}}}
$$

where $\chi=\xi_{1} /\left(\pi f_{i}^{\prime} T_{s}\right)$.

As an application of the previous analysis, let assume that the resonator in Fig.6 is formed by fully-differential regulated-folded cascode memory cells like that shown in Fig.7. Because of the input feedback loop (which increases the input conductance), this memory cell exhibits a third-order dynamics. It can be shown that the current source named $I_{b r}$ (see Fig.7) has to be taken as large as possible in order to obtain an overdamped settling response. However, large values of $I_{b r}$ may force some transistors to leave the saturation region, thus causing a non-linear dependence of the input voltage on the input signal. This behavior can be modeled as

$$
V_{i d}=r_{1} I_{D}+r_{3} I_{D}^{3}
$$

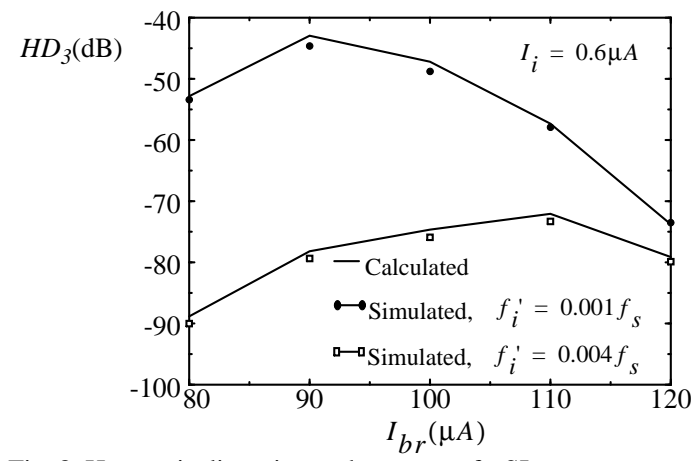

Fig. 8: Harmonic distortion at the output of a SI resonator caused by the non-linear input impedance of regulated folded cascode memory cells.

where $V_{i d}$ and $I_{D}$ are respectively the differential input voltage and the differential drain current of each memory cell. Coefficients $r_{1}$ and $r_{3}$, which are function of $I_{b r}$, were extracted from DC nominal HSPICE simulations. It can be shown that

$$
\xi_{1} \cong-2 g_{\text {out }} r_{1} \quad \xi_{3} \cong-2 g_{\text {out }} r_{3}
$$

where $g_{\text {out }}$ is the output conductance of the memory cell. Thus, the harmonic distortion of the resonator can be calculated by simply substituting (37) in (35).

Fig. 8 compares theoretical results with simulations by plotting $\mathrm{HD}_{3}$ as a function of $I_{b r}$ for different values of $f_{i}^{\prime}$. Note that, as a consequence of changing both the linear and the non-linear error, the harmonic distortion does not increase with $I_{b r}$. This is well predicted by the model. Because of the resonator open loop gain is too high, the input amplitude was chosen to be small $\left(I_{i}=0.6 \mu A\right)$ in order to keep memory transistors in the saturation region.

\section{B. Harmonic distortion in SI 4th-order bandpass $\Sigma \Delta$ modulators}

Fig.9 shows the block diagram of a fourth-order bandpass $\Sigma \Delta$ modulator (4thBP $\Sigma \Delta \mathrm{M}$ ) based on LDI loop resonators like that shown in Fig.6. Modelling the quantizer as an additive white noise source $E(z)$ and considering that the integrators are ideal, the $z$-domain equations that describe the behavior of the modulator are:

$$
\begin{gathered}
Y(z)=z^{-2} X(z)+\left(1+z^{-2}\right)^{2} E(z) \\
I_{1}(z)=X(z)-z^{-2} Y(z)-2 I_{o}(z) \\
I_{2}(z)=\frac{-z^{-1 / 2}}{\left(1-z^{-1}\right)} I_{1}(z) \\
I_{o}=\frac{z^{-1}}{1+z^{-2}}\left(X(z)-z^{-2} Y(z)\right)
\end{gathered}
$$

where $X(z)$ and $Y(z)$ represents respectively the input and the output of the modulator; $I_{1}(z)$ and $I_{2}(z)$ are the input and the output of the first integrator; $I_{i}(z)$ and $I_{o}(z)$ are the input and the output of the first resonator.

Only the contribution of the first resonator will be 


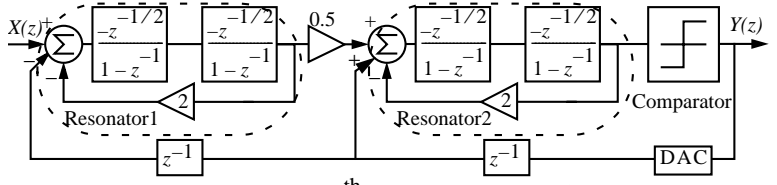

Fig. 9: Block diagram of the $4^{\text {th }}$-order bandpass $\Sigma \Delta$ modulator.

considered for the analysis of the distortion. This is because the contribution of the second resonator is attenuated by the gain of the first resonator in the signal band.

From (38), the amplitude of the resonator output can be written as

$$
I_{o}=\left.A\left|1-z^{-2}\right|\right|_{z=e^{j 2 \pi f_{i} T_{s}}}
$$

where $A$ is the amplitude of the modulator input. Taking into account the effect of the linear gain error $\xi_{1}$ on the integrators, it can be shown that the above expression is modified as follows:

$$
\left.I_{o} \cong A\left(1-6 \xi_{1}\right)\left|1-z^{-2}\right|\right|_{z=e^{j 2 \pi f_{i} T_{s}}}
$$

Substituting (40) in (34) and dividing by $A$ the third-order harmonic distortion at the modulator output is approximately given by

$$
H D_{3} \cong 4 \xi_{3} A^{2}\left(1-16 \xi_{1}\right)\left(1+12 \pi f_{i}^{\prime} T_{s}\right)
$$

In bandpass signal processing, the third-order intermodulation distortion, $I M_{3}$ is more appropriate for measuring distortion than $H D_{3}$. Let assume that the modulator input consists of two sinusoidal signals of the same amplitude and different frequencies $f_{1}$ and $f_{2}$. If the memory cells which form the modulator are degraded by non-linear errors, the modulator output spectrum presents intermodulation harmonics of the input signals. Among them, the most significant is $I M_{3}$, which is defined as the amplitude of the output at $2 f_{2}-f_{1}$ and $2 f_{1}-f_{2}$ related to the linear output amplitudes at $f_{2}, f_{1}$. It can be shown that $I M_{3}$ is related to $\mathrm{HD}_{3}$ as

$$
I M_{3}=3 H D_{3}
$$

For illustration, Fig.10(a) compares simulation results to theoretical data by plotting $I M_{3}$ against $I_{b r}$ for different values of the DAC reference current $I_{D A C}$. Fig.10(b) shows the output spectrum of the modulator for $I_{b r}=110 \mu \mathrm{A}$ and $I_{D A C}=50 \mu \mathrm{A}$. The predicted value for $I M_{3}$ is $-53 \mathrm{~dB}$ which agrees with the simulated value $(-51 \mathrm{~dB})$

\section{Conclusions}

The impact of main SI errors on the harmonic distortion in both lowpass and bandpass $\Sigma \Delta$ modulators has been analysed. Closed form equations for the thirdorder harmonic distortion and the third-order intermodulation distortion of their main blocks are also provided. General expressions are derived which can be particularized for each SI error. All results are validated by time-domain behavioral simulations [11].
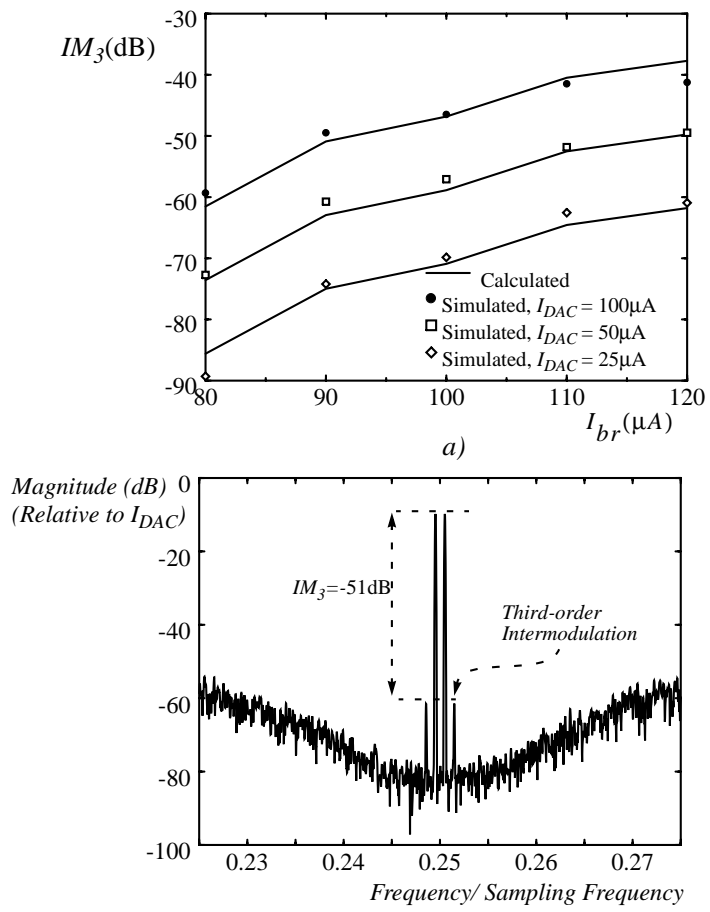

b)

Fig. 10: $I M_{3}$ at the output of a $4^{\text {th }}$ order bandpass $\Sigma \Delta$ modulator due to the non-linear input impedance of regulated folded-cascode memory cells. a) $I M_{3}$ vs. $I_{b r}$. b) Output spectrum corresponding to $I_{b r}=110 \mu \mathrm{A}$, and $I_{P A C}=50 \mu \mathrm{A}$. (Amplitude of each input tone equal to $\left.(1 / 2 \sqrt{2}) I_{D A C}\right)$.

\section{References}

[1] C.Toumazou, J.B.Hughes, and N.C. Battersby, Switched-Currents: An Analogue Technique for digital technology, London, Peter Peregrinus Ltd.,1993.

[2] J.B. Hughes, K.W. Moulding, J. Richardson, J. Bennet, W. Redman-White, Mark Bracey and R. Singh Soin, "Automated Design of Switched-Current Filters", IEEE J. Solid-State Circuits, pp. 898-907, July 1996.

[3] B.E. Jonsson and H. Tenhunen, "Low-Voltage, 10bit Switched-Current ADC with $20 \mathrm{MHz}$ Input Bandwidth", Electron. Lett.,pp. 1904-1905,October 1998.

[4] N.Tan, Switched-Current Design and Implementation of Oversampling A/D Converters, Kluwer Academic Publishers, 1997.

[5] N. Moeneclaey and A. Kaiser, "Design Techniques for High-Resolution Current-Mode Sigma-Delta Modulators", IEEE Journal of Solid-State Circuits, pp. 953-958, July 1997.

[6] J.M. de la Rosa, B. Pérez-Verdú, F. Medeiro and A Rodríguez-Vázquez, "A $2.5 \mathrm{MHz} 55 \mathrm{~dB}$ Switched-Current BandPass $\Sigma \Delta$ Modulator for AM Signal Conversion", Proc. of the 1997 European Solid-State Cicuits Conference, pp. 156-159, 1997.

[7] F. Medeiro, B. Pérez-Verdú and A. Rodríguez-Vázquez, Top-Down Design of High-Performance Sigma-Delta Modulators, Kluwer Academic Publishers, 1999.

[8] T.S. Fiez, G. Liang, and D.J. Allstot, "Switched-Current Circuit Design Issues", IEEE J. Solid-State Circuits, pp. 192-202, March 1991.

[9] J.M. Martins and V. F. Dias, "Harmonic Distortion in Switched-Current Audio Memory Cells", IEEE Transactions on Circuits and Systems- Part II,Vol. 46, pp. 326-334, March 1999.

[10]J.M. Martins, V.F. Dias, "Harmonic Distortion in Switched-Current Sigma-Delta Modulators due to Clock Feedthrough", Proc. 1998 IEEE Int. Symp. Circuits and System, 1998.

[11]J.M. de la Rosa, A. Kaiser and B. Pérez-Verdú, "Interactive Verification of Switched-Current Sigma- Delta Modulators", Proc. of 1998 IEEE International Conference on Electronics, Circuits and Systems, pp. 2.157-2.160, 1998.

[12]S.R. Norsworthy, R. Schreier, G.C. Temes: Delta-Sigma Converters. Theory, Design and Simulation. New York, IEEE Press, 1997. 